\title{
Enhancement of Nematic Order of Liquid Crystals in Thin Cells
}

\author{
P. ASWInI ${ }^{a, b}$ AND A.S. GOVInD ${ }^{a, *}$ \\ ${ }^{a}$ Department of Physics, Vijaya College, R.V. Road, Basavanagudi, Bangalore - 560004 India \\ ${ }^{b}$ MGR Educational and Research Institute, Deemed University, Chennai, India
}

(Received November 24, 2011)

\begin{abstract}
Experimental observations show that the nematic order parameter is significantly enhanced as the thickness of the cell is reduced. Calculations of earlier theories do not agree well with the experimental data. We propose a simple extension of Maier-Saupe theory to account for the enhancement. A molecule near the surface is assumed to feel the mean field potential (the Maier-Saupe type) and also the surface induced potential. This is included in the Maier-Saupe theory. Our calculations are in better agreement with the experimental data.
\end{abstract}

PACS: 61.30.Gd, 61.30.-v, 64.70.mf

\section{Introduction}

Nematic liquid crystals have a long-range orientational order of the long axes of the molecules [1]. The orientational order parameter is given by $S=\left\langle P_{2} \cos \theta\right\rangle$. Studies of liquid crystal (LC) in confined geometries are particularly very important both from fundamental and technological point of view. LCs are used in display devices. The typical thickness used in bistable displays is about $2 \mu \mathrm{m}$. Twisted nematic LC is widely used in many display systems. The $d / p$ ratio affects the switching time, where $d$ is the cell thickness and $p$ is the pitch. The ratio $d / p$ shows the deviation in thin cells. Such deviation is observed to increase as the cell thickness decreases and can be appreciable for $d \leq 7 \mu \mathrm{m}$. It is found that the order parameter at the surface of the substrate as well as in the interior of the sample increases with decreasing cell thickness. There are several theoretical and experimental studies to show that the enhancement is larger as the thickness is reduced [2-8]. We discuss this significant enhancement of birefringence $\Delta n(\propto S$, where $S$ is the orientational order parameter) in thin cells.

Assuming a perfect orientational order at the surface, calculations based on the Landau-de Gennes theory show that the thickness averaged enhancement of $S$ increases as the temperature is close to the nematic-isotropic transition temperature [9]. The measured order parameter $S$ is further enhanced in thin cells because of the stiffening of the elastic constant. This reduces the thermal fluctuations of the nematic director. The combined effect is too small at low temperatures to account for the experimental data [10]. We propose a simple extension of the Maier-Saupe (M-S) theory to account for the enhancement.

In our model, a molecule near the surface is assumed to feel the mean field potential (M-S type) and also sur-

* corresponding author; e-mail: govindas63@gmail.com face induced potential. This is included in M-S theory. Our calculations are in better agreement with the experimental data [10].

\section{Theoretical model}

The distance from the surface of the cell into the medium is taken along the $z$-axis. The medium is assumed to be made up of layers of thickness $\mathrm{d} z$ parallel to the cell wall. All calculations are made for a layer and then integrated over $z$. $U_{i}$ is the molecular mean field potential of $i$-th molecule at $z$. $S_{z}$ is the order parameter for molecules in the layer between $z$ and $z+\mathrm{d} z$. $F / N$ is the average Helmholtz free energy per molecule in the layer between $z$ and $z+\mathrm{d} z$.

A molecule at $z$ feels the mean field potential (M-S type) and also surface induced potential. The exact nature of the variation of surface potential with respect to $z$ is not known experimentally. The potential has the maximum at $z=0$ and zero at large distances from the surface. Thus, the surface induced potential is taken empirically to decay exponentially as

$$
U_{i}=U_{\mathrm{s}} \mathrm{e}^{-z / r_{0}},
$$

where $U_{\mathrm{s}}$ is surface potential at $z=0$ and $r_{0}$ is the characteristic length. The layer thickness is taken to be quite small, comparable to molecular length.

The mean field is not provided by the bulk medium since the interaction beyond few layers is negligible. Also the effect of gradient $\mathrm{d} S / \mathrm{d} z$ and its elastic energy effects are shown to be very small [10]. Hence, also for M-S type mean field, we use $S$ instead of $S_{z}$. Variation in the density of the medium due to the variation of $S$ is ignored.

The order parameter, averaged over the full thickness of the cell is

$$
\langle S\rangle=\frac{\int_{0}^{t / 2} S_{z} \mathrm{~d} z}{\int_{0}^{t / 2} \mathrm{~d} z},
$$

where $t$ is the cell thickness. The integration is done up to half the cell thickness since the other half is symmetric. 
This is to be compared with the experimental values of $\langle S\rangle$.

Surface potential at $z=0$ is $U_{\mathrm{s}}=A U_{0}$ where $A$ is a constant and $U_{0}$ is the constant in $\mathrm{M}-\mathrm{S}$ theory with

$$
\frac{U}{k T_{\mathrm{NI}}}=4.541 \text {, }
$$

$k$ being the Boltzmann constant and $T_{\mathrm{NI}}$ - the nematicisotropic transition temperature.

\section{Expression for free energy and order parameter}

As explained above, the potential of the $i$-th molecule at $z$ is written as

$$
U_{i}=-U_{0} S P_{2}\left(\cos \theta_{i}\right),
$$

where $P_{2}$ is the 2 nd Legendre polynomial and $\theta_{i}$ is the angle between nematic director and the long axis of the $i$-th molecule.

The average internal energy per molecule is

$$
\frac{U}{N}=\frac{U_{0} S^{2}}{2}-U_{\mathrm{s}} \mathrm{e}^{-z / r_{0}} S,
$$

where $N$ is the Avogadro number and $S=\left\langle P_{2}\left(\cos \theta_{i}\right)\right\rangle$ is the order parameter at $z$, with \langle\rangle representing the statistical average.

The molar entropy is $\Im=N k\langle\ln f\rangle$ where $f$ is the probability distribution function.

The average Helmholtz free energy per molecule is

$$
\frac{F}{N k T}=-\frac{U_{0} S^{2}}{2 k T}-\frac{U_{\mathrm{s}}}{k T} \mathrm{e}^{-z / r_{0}} S+\langle\ln f\rangle,
$$

where $\langle\ln f\rangle=\int_{0}^{1} f \ln f \mathrm{~d} x$ with $x=\cos \theta$.

Hence the order parameter is

$$
S=\int_{0}^{1} f P_{2}(x) \mathrm{d} x,
$$

minimizing $F$ with respect to (w.r.t.) $f$ we get

$$
f=\frac{\exp \left(\frac{U_{0}}{k T}\left(S+A \mathrm{e}^{-z / r_{0}}\right) P_{2}(x)\right)}{Z},
$$

where $Z=\int_{0}^{1} f \mathrm{~d} x$ is the partition function.

Substituting for $\langle f \ln f\rangle$ we get

$$
\frac{F}{N k T}=\frac{U_{0} S^{2}}{2 k T}-\ln Z \text {. }
$$

Minimizing $F$ w.r.t. $S$ we get

$$
S=\int f P_{2}(x) \mathrm{d} x,
$$

which satisfies the self-consistency condition.

\section{Method of calculation}

The necessary integrals are evaluated numerically using 32 point Gaussian quadrature method in double precision. Numerical iteration is used to adjust $S$ for self-consistency. The constant $A$ is taken to be 10 as in [9]. After calculating $S$ at different values of $z$, the average value $\langle S\rangle$ is found for a given thickness $(t)$ using Eq. (2). This is repeated for different values of $t$ and also for thicknesses $1 \mu \mathrm{m}$ and $10 \mu \mathrm{m}$, respectively. In each case the percentage change in nematic order parameter $\mathrm{d} S_{1} \%, \mathrm{~d} S_{2} \%$ and $\mathrm{d} S \%$ are calculated using

$$
\begin{gathered}
\mathrm{d} S \%=\frac{\langle S\rangle_{1 \mu \mathrm{m}}-\langle S\rangle_{10 \mu \mathrm{m}}}{\langle S\rangle_{10 \mu \mathrm{m}}} \times 100, \\
\mathrm{~d} S \%_{1 \mu \mathrm{m}}=\frac{\langle S\rangle_{1 \mu \mathrm{m}}-\langle S\rangle_{\mathrm{b}}}{\langle S\rangle_{\mathrm{b}}} \times 100, \\
\mathrm{~d} S \%_{10 \mu \mathrm{m}}=\frac{\langle S\rangle_{10 \mu \mathrm{m}}-\langle S\rangle_{\mathrm{b}}}{\langle S\rangle_{\mathrm{b}}} \times 100,
\end{gathered}
$$

where $S_{\mathrm{b}}$ is the bulk value, corresponding to large value of $z$ at which $U_{\mathrm{s}}$ has become negligible.

We have tried for another surface induced potential with inverse square dependence of the type

$$
U_{i}=U_{\mathrm{s}} \frac{1}{1+\left(z / r_{0}\right)^{2}}
$$

with $U_{\mathrm{s}}=U_{0} A$ as earlier.

Minimising the free energy per molecule we get

$$
f=\frac{\exp \left(\frac{U_{0}}{k T}\left(S+A \frac{1}{1+\left(z / r_{0}\right)^{2}}\right) P_{2}(x)\right)}{Z} .
$$

The value of $\langle S\rangle$ is found for different values of $T$, for thicknesses $1 \mu \mathrm{m}$ and $10 \mu \mathrm{m}$, respectively, as earlier.

\section{Results and discussion}

Calculated variations of order parameter $\left(S_{Z}\right)$ with distance from surface $(z)$ for a cell of thickness $1 \mu \mathrm{m}$ and $10 \mu \mathrm{m}$, at $T=T_{\mathrm{NI}}-1^{\circ}$ and $T=T_{\mathrm{NI}}-10^{\circ}$ are shown below in Figs 1 and 2. The variations are plotted up to a middle of cell since the other half is symmetric. The $X$-axis is taken as $z / r_{0}$, with $r_{0}=0.01 \mu \mathrm{m}$.

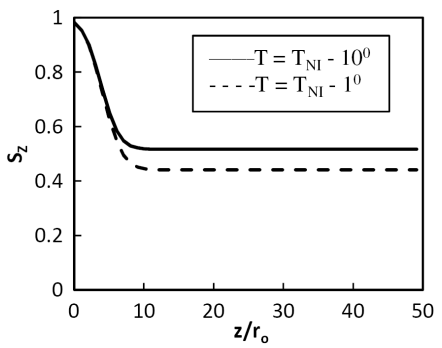

Fig. 1. Variation of order parameter $S_{z}$ with distance from surface $z$ for a cell of thickness $1 \mu \mathrm{m}$, up to middle of cell, with $r_{0}=0.01 \mu \mathrm{m}$, at $t=T_{\mathrm{NI}}-1^{\circ}$ and $T_{\mathrm{NI}}-10^{\circ}$.

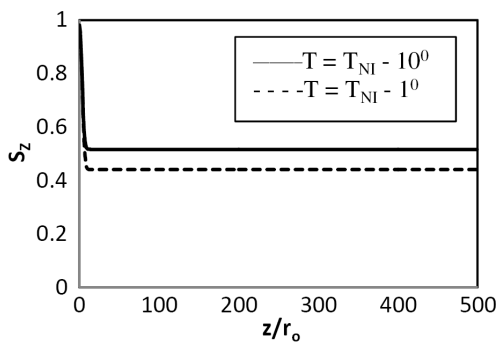

Fig. 2. Variation of order parameter $S_{z}$ with distance from surface $z$ for a cell of thickness $10 \mu \mathrm{m}$, up to middle of cell, with $r_{0}=0.01 \mu \mathrm{m}$, at $t=T_{\mathrm{NI}}-1^{\circ}$ and $T_{\mathrm{NI}}-10^{\circ}$. 
Note that the $S_{z}$ at the surface is nearly 0.98 since the surface potential at $z=0$ is strong. It decreases to the bulk value away from the surface.

Tables I and II show the variation of $S_{\mathrm{b}}$ and $\mathrm{d} S \%$ at different temperatures near $\mathrm{T}_{\mathrm{NI}}$. The experimental values are as reported in Ref. [10]. It can be seen that, close to $\mathrm{T}_{\mathrm{NI}}$, the enhancement is large and decreases as the temperature is lowered. This trend is expected, as the surface potential has a larger effect in increasing the order parameter when the bulk order parameter is lower. The bulk order parameter increases with the decrease in temperature and the effect of surface order is reduced.

Experimentally it is found [10] that the enhancement is not significantly reduced at lower temperatures. Consid- ering the first potential (exponential decay) the percentage enhancement of the order parameter between thick and thin cells reduces by 1.5 times with the decrease in temperature, while for the second potential (inverse square law) the reduction is 1.7 times. However, the reduction as per the calculations in Ref. [10] is 50 times. Thus, the simple empirical equations used for the decay of surface potential give results which are in better agreement with experimental data compared to earlier theories [10]. Of the two potentials used, the second potential is better than the first as seen in Table III. This gives an insight about the realistic variation of surface effects which may be investigated more rigorously.

TABLE I

Comparison of calculated values of order parameters $(S)$ in cells of thicknesses $1 \mu \mathrm{m}$ and $10 \mu \mathrm{m}$ for the first potential (exponential decay).

\begin{tabular}{c|c|c|c|c|c|c|c|c}
\hline \hline Temperature & $S_{\mathrm{b}}[10]$ & $S_{\mathrm{b}}$ & $\left\langle S_{1}\right\rangle$ & $\left\langle S_{2}\right\rangle$ & $\mathrm{d} S_{1} \%$ & $\mathrm{~d} S_{2} \%$ & $\mathrm{~d} S \%$ & $\mathrm{~d} S \%[10]$ \\
\hline$T_{\mathrm{NI}}-0.2^{\circ}$ & 0.297 & 0.4311 & 0.4835 & 0.4363 & $12.15 \%$ & $1.21 \%$ & $10.8 \%$ & $30 \%$ \\
$T_{\mathrm{NI}}-1^{\circ}$ & 0.373 & 0.4407 & 0.4918 & 0.4458 & $11.58 \%$ & $1.16 \%$ & $10.3 \%$ & $9 \%$ \\
$T_{\mathrm{NI}}-5^{\circ}$ & 0.566 & 0.4799 & 0.5257 & 0.4845 & $9.55 \%$ & $0.96 \%$ & $8.5 \%$ & $1.5 \%$ \\
$T_{\mathrm{NI}}-10^{\circ}$ & 0.714 & 0.5165 & 0.5578 & 0.5206 & $8 \%$ & $0.8 \%$ & $7.1 \%$ & $0.6 \%$
\end{tabular}

TABLE II

Comparison of calculated values of order parameters $(S)$ in cells of thicknesses $1 \mu \mathrm{m}$ and $10 \mu \mathrm{m}$ for the second potential (inverse square type).

\begin{tabular}{c|c|c|c|c|c|c|c|c}
\hline \hline Temperature & $S_{\mathrm{b}}[10]$ & $S_{\mathrm{b}}$ & $\left\langle S_{1}\right\rangle$ & $\left\langle S_{2}\right\rangle$ & $\mathrm{d} S_{1} \%$ & $\mathrm{~d} S_{2} \%$ & $\mathrm{~d} S \%$ & $\mathrm{~d} S \%[10]$ \\
\hline$T_{\mathrm{NI}}-0.2^{\circ}$ & 0.297 & 0.4311 & 0.5136 & 0.4403 & $16.67 \%$ & $2.11 \%$ & $16.49 \%$ & $30 \%$ \\
$T_{\mathrm{NI}}-1^{\circ}$ & 0.373 & 0.4407 & 0.5192 & 0.4494 & $15.66 \%$ & $1.95 \%$ & $15.53 \%$ & $9 \%$ \\
$T_{\mathrm{NI}}-5^{\circ}$ & 0.566 & 0.4799 & 0.5257 & 0.4871 & $12.36 \%$ & $1.479 \%$ & $12 \%$ & $1.5 \%$ \\
$T_{\mathrm{NI}}-10^{\circ}$ & 0.714 & 0.5165 & 0.5726 & 0.5225 & $10 \%$ & $1.142 \%$ & $9.6 \%$ & $0.6 \%$
\end{tabular}

TABLE III

Comparison of $\mathrm{d} S \%$ in first and second potentials.

\begin{tabular}{c|c|c|c|c}
\hline \hline Compound [10] & Thickness of the thin and thick cells $[\mu \mathrm{m}]$ & $\mathrm{d} S \%[10]$ & $\mathrm{d} S \%$ (first potential) & $\mathrm{d} S \%$ (second potential) \\
\hline S1014 & 1.5 and 6.7 & $15 \%$ & $3.85 \%$ & $10.10 \%$ \\
CN & 1.5 and 14 & $11 \%$ & $4.26 \%$ & $12.58 \%$
\end{tabular}

Due to increased orientational order, the smectic phase may be stabilised near the surface. In fact, experiment [9] shows that layers of smectic are possible near the surface. This can be discussed by including the surface potential to extend McMillan theory for the smectic phase. Further work in this direction is under progress.

\section{Conclusions}

Experimental observations show that the nematic order parameter is significantly enhanced as the thickness of the cell is reduced and that this enhancement does not significantly reduce as the temperature is lowered below $T_{\mathrm{NI}}$. Calculations of earlier theories do not agree well with the experimental data. We use an empirical variation of surface potential and have proposed the simple extension of the Maier-Saupe theory to account for the enhancement. Our results are in better agreement with experimental results.

\section{References}

[1] P.G. de Gennes, J. Prost, The Physics of Liquid Crystals, 2nd ed., Clarendon Press, Oxford 1993.

[2] T.Z. Qian, Z.L. Xie, H.S. Kwok, P. Sheng, Appl. Phys. Lett. 71, 596 (1997).

[3] P. Sheng, Phys. Rev. A 26, 1610 (1982).

[4] P. Sheng, Phys. Rev. Lett. 37, 1059 (1976).

[5] K. Miyano, Phys. Rev. Lett. 43, 51 (1979).

[6] H. Mada, S. Kobayashi, Appl. Phys. Lett. 35, 4 (1979).

[7] R.W. Sobha, D. Vijayaraghavan, N.V. Madhusudhana, Europhys. Lett. 44, 296 (1998).

[8] I. Haller, Prog. Solid State Chem. 10, 103 (1975).

[9] J.V. Selinger, D.R. Nelson, Phys. Rev. A 37, 1736 (1988).

[10] S. Dhara, N.V. Madhusudhana, Europhys. J. E 13, 401 (2004). 